\title{
A Dose Meter For Pulsed Neutron Fields
}

\author{
Alfred Klett' ${ }^{1}$ Albrecht Leuschner², Norbert Tesch² \\ 1) Berthold Technologies GmbH \& Co KG, Calmbacherstrasse 22, 75323 Bad Wildbad, Germany \\ 2) Deutsches Elektronen-Synchrotron DESY, Notkestrasse 85, 22607 Hamburg, Germany
}

\section{Introduction}

A new dose meter for the measurement of continuous and pulsed neutron and gamma radiation fields was recently developed by DESY, Berthold Technologies and Struck Innovative Systems [1]. The instrument is intended for high energy applications at accelerators. The concept for pulsed radiation measurement is based on activation of shortlived radionuclides in the detector materials or in the detector surroundings.

\section{The Detection System}

The instrument comprises two different and independent radiation detection channels

- ${ }^{3}$ He proportional counter tube in a moderator for neutron detection

- Plastic scintillator with PMT for beta-gamma detection

The system is capable of both gamma and neutron area dose measurement in

- continuous radiation fields

- pulsed radiation fields

Fast readout and analysis of the detector signals as well as Ethernet communication with external hosts is provided by a flash-ADC-board designed by Struck. While measurement in continuous fields is conventional there are in pulsed fields several reactions with ${ }^{12} \mathrm{C}$ as target nucleus and the production of $8 \mathrm{Li}$, $9 \mathrm{Li}$ and ${ }^{12} \mathrm{~B}$ (with respective halflives 840 , 170 and $20 \mathrm{~ms}$ ) utilized. The two measuring channels are detecting decay particles from the instable nuclides with a precise time-stamp. The system auto-synchronizes with the time structure of the pulsed radiation field and accumulates time distributions of the recorded events relative to the beam pulses. The delayed decay products are identified by analyzing their timing characteristics. From these data dose results not affected by dead time can be extracted. Details have been reported earlier [1], [2]. Since the end of 2008 at PETRA III DESY there are already 30 units in operation [3]. The integrated system will be available on the market as BERTHOLD LB 6419.

\section{New Calibration Results}

\section{Neutron Dose Response}

LB 6419 calibration measurements in unpulsed continuous neutron radiation fields were during August-September 2009 performed at National Physical Laboratories NPL in Teddington/UK. We used neutrons generated by the neutron sources ${ }^{241} \mathrm{Am}-\mathrm{Be},{ }^{252} \mathrm{Cf}$ and $\mathrm{D}_{2} \mathrm{O}$ moderated ${ }^{252} \mathrm{Cf}$ and by the accelerator for the thermal column and for monoenergetic neutrons at $2.5 \mathrm{MeV}$ and $17 \mathrm{MeV}$. The LB 6419's measured dose responses to $\mathrm{H}^{*}(10)$ are shown together with the results of a FLUKA calculation in Fig. 4. These data are related to the instrument's reference orientation perpendicular to the principal axis and were calculated with the conversion factors of ICRP74.

\section{Photon Dose Response}

The LB 6419's response to unpulsed continuous photon dose was calibrated at several photon energies at the irradiation facilities of the Eichamt München-Traunstein / Verification Office in Munich Germany. The measurements were performed between 3-4 March 2009 where we used the ISO photon radiation qualities N-20, N-40, N-60, N-80, N-100, $\mathrm{N}-120, \mathrm{~N}-150, \mathrm{~N}-200, \mathrm{~N}-300$ and the gamma sources ${ }^{137} \mathrm{Cs}$ and ${ }^{60} \mathrm{Co}$. The mean energies of these radiation fields are $17,33,48,65,83,100,118,161,248,662$ and $1200 \mathrm{keV}$ respectively covering a wide range. The plastic scintillator's photon dose response as a function of energy is shown in fig. 5. These data are also related to the reference orientation perpendicular to the principal axis.

\section{References}

[1] Klett, Alfred and Leuschner, Albrecht : A Pulsed Neutron Monitor, IEEE 2007 Nuclear Science Symposium and Medical Imaging Conference, Conference Records, October 27- November 3, 2007, Honolulu, Hawaii, USA

[2] A. Leuschner, N. Tesch, W. Clement, K.P. Klimek, M. Lomperski, M. Sachwitz, M. Morgenstern, A. Klett, M. Kirsch, T. Häupke, The Radiation Monitor PANDORA (LB 6419) at PETRA III, The $5^{\text {th }}$ International Workshop on Radiation Safety at Synchrotron Radiation Sources, RadSynch'09, Trieste, Italy, May 21-23, 2009 Conference Proceedings will be published in Radiation Measurements, Special Issue, Elsevier

[3] PETRA III: A Low Emittance Synchroton Radiation Source, TDR 2004, ISSN 0418-9833.

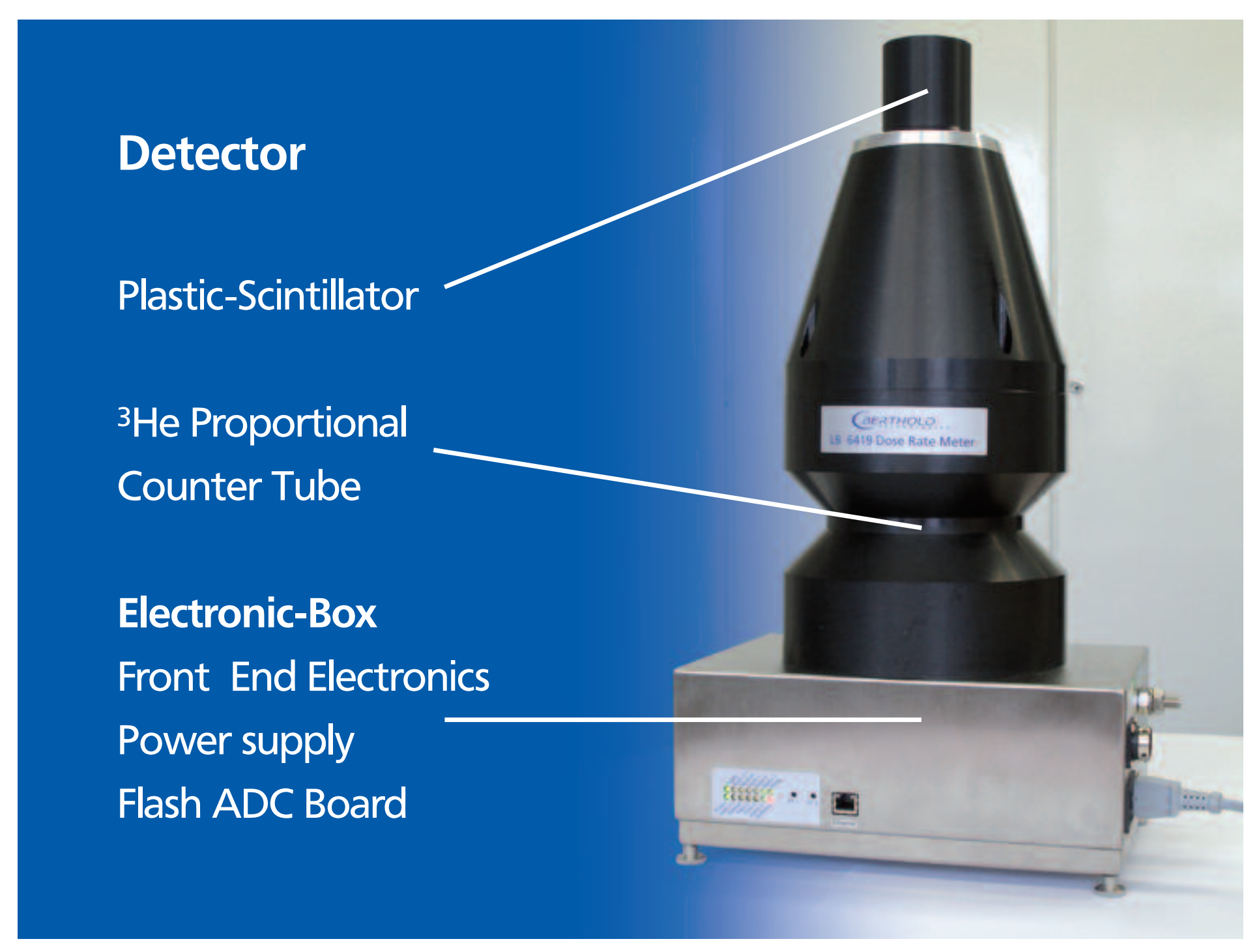

Figure 1: LB 6419 Dose Meter

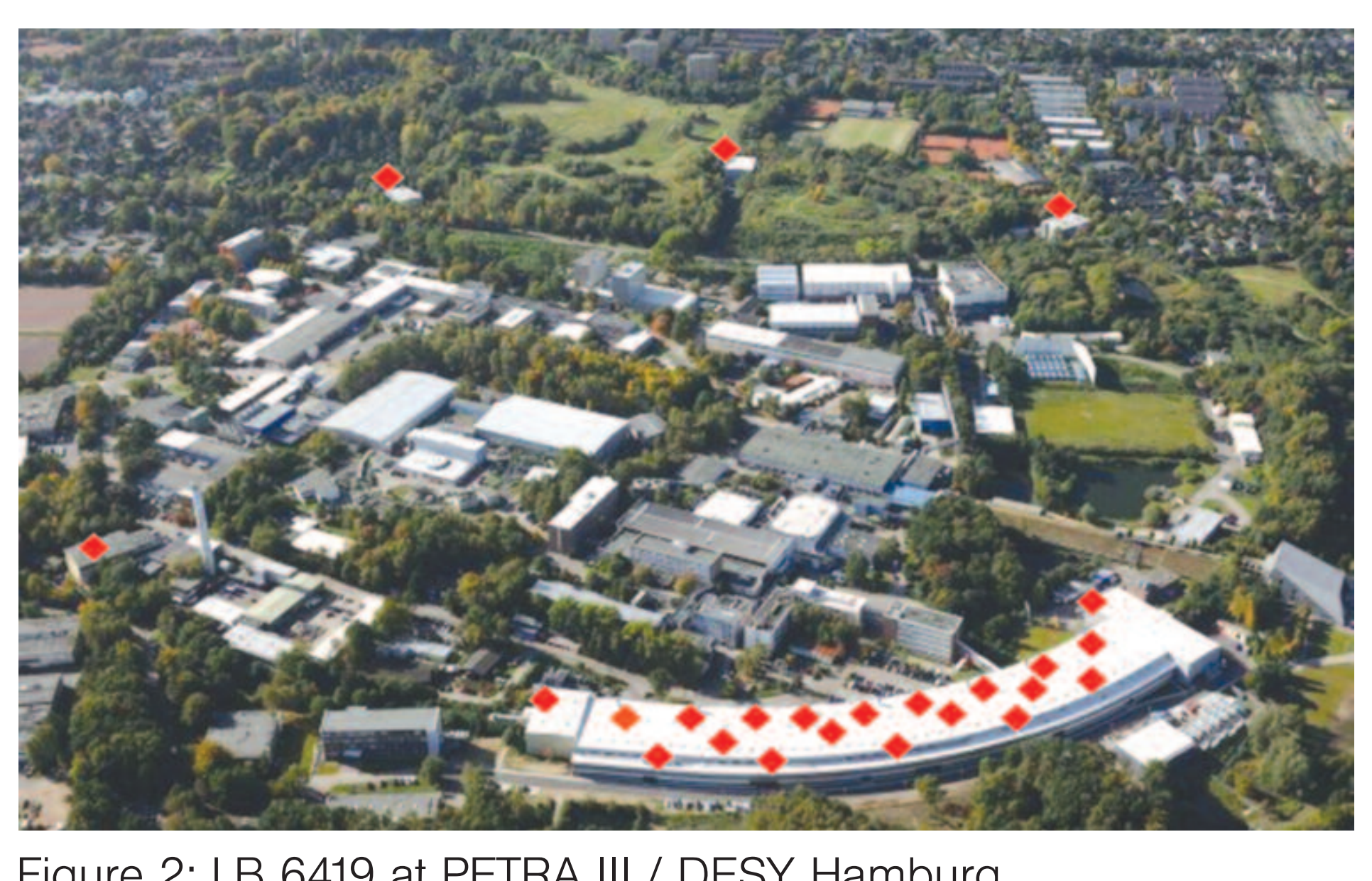

Figure 2: LB 6419 at PETRA III / DESY Hamburg

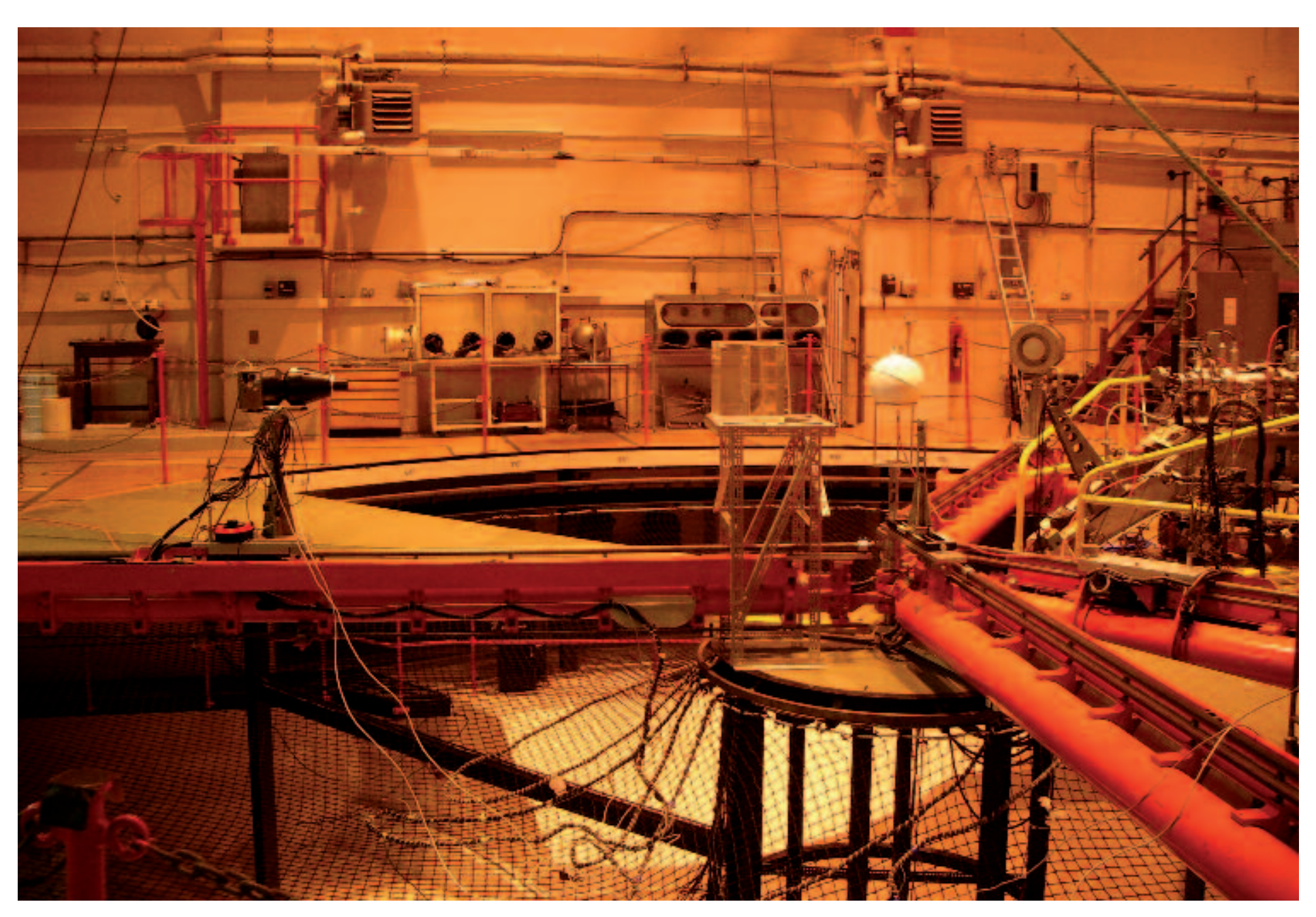

Figure 3: NPL $\mathrm{D}_{2} \mathrm{O}$ moderated ${ }^{252} \mathrm{Cf}$ calibration

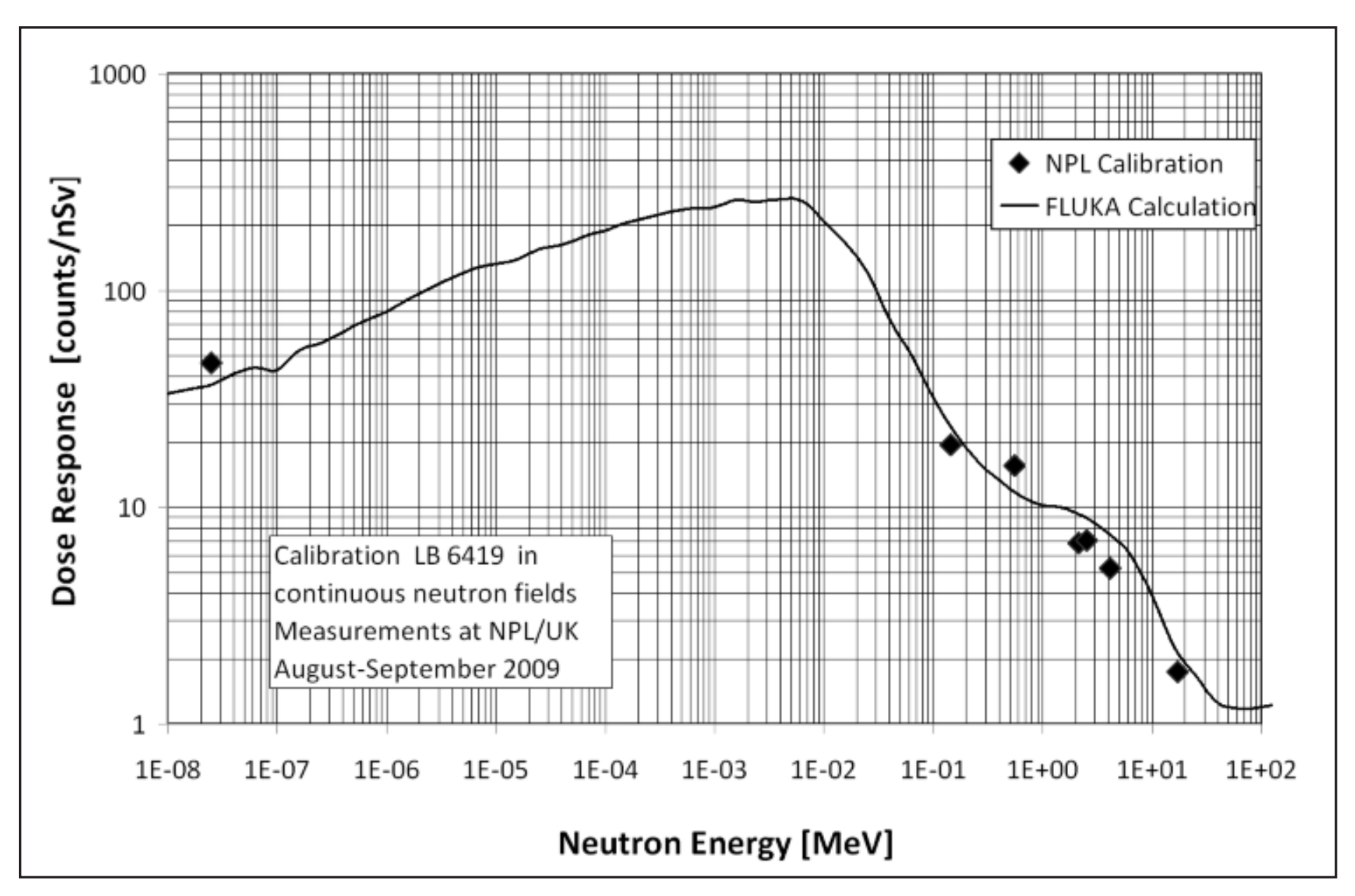

Figure 4: Neutron Dose Response

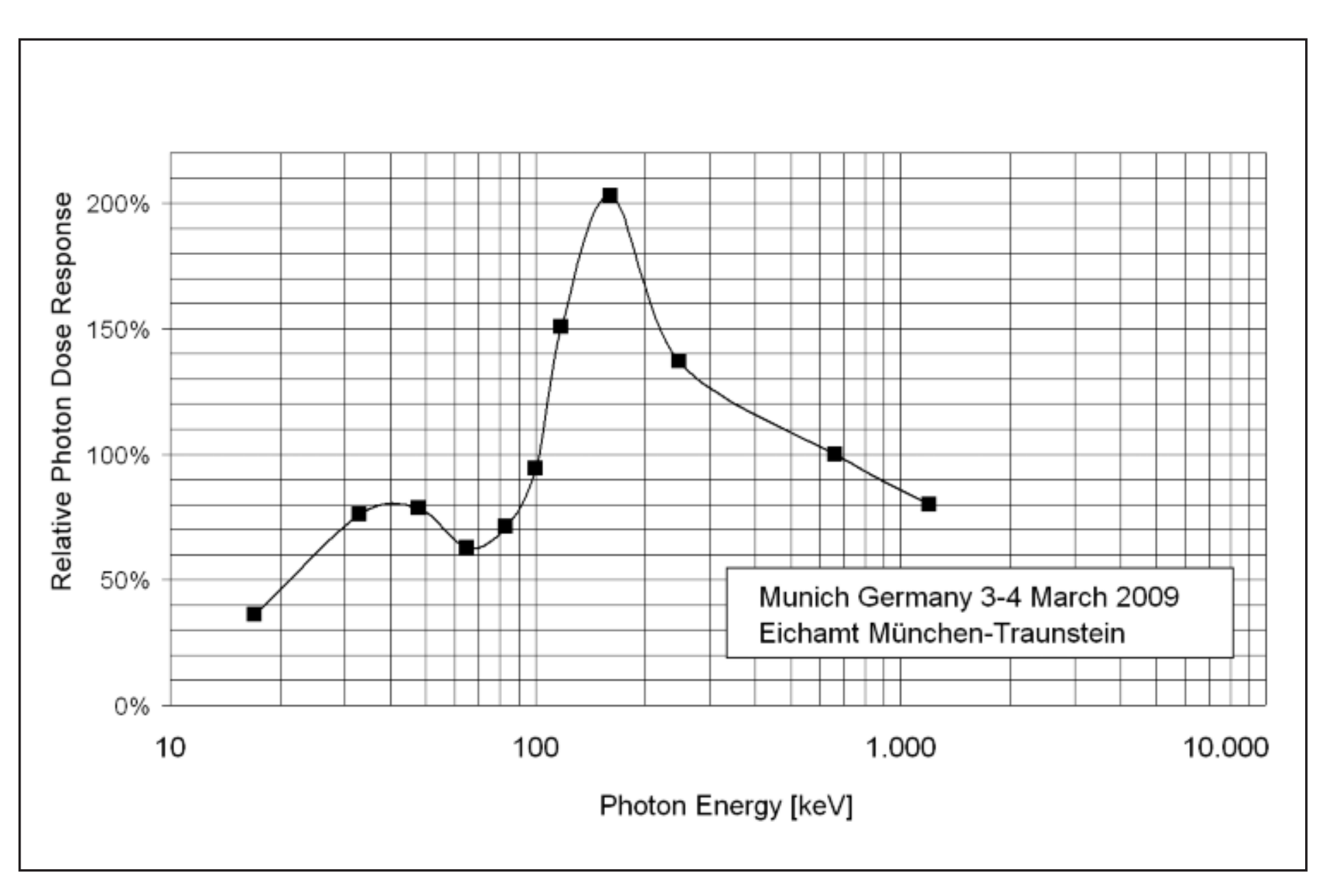

Figure 5: Photon Dose Response 